\title{
Novel SSR and SNP markers in Viola yedoensis Makino resistant to cadmium stress
}

\author{
Hua Peng ${ }^{\mathrm{a}, \uparrow}$, Jian Gao ${ }^{\mathrm{b}, \mathrm{c}, \dagger}$, Chun Zhang ${ }^{\mathrm{d}, *}$, Lilan Ou ${ }^{\mathrm{d}}$, Mao Luo ${ }^{\mathrm{a}, \mathrm{d}, \mathrm{e}, *}$ \\ a Department of Tourism and Cultural Industry, Sichuan Tourism College, Chengdu 610100 China \\ ${ }^{b}$ Department of Life Sciences and Technology, Yangtze Normal University, Fuling 408100 China \\ c Center for Green Development and Collaborative Innovation in Wuling Mountain Region, Yangtze \\ Normal University, Fuling 408100 China \\ d Department of Pharmacology, School of Pharmacy, Southwest Medical University, Luzhou 646000 China \\ e Collaborative Innovation Center for Prevention and Treatment of Cardiovascular Disease of Sichuan \\ Province, Drug Discovery Reseach Center, Southwest Medical University, Luzhou 646000 China
}

*Corresponding authors, e-mail: zc83good@126.com, luomao20050908@163.com, Peng H. and Gao J. contributed equally to this article

Received 10 Jul 2019

Accepted 1 Apr 2020

\begin{abstract}
Viola yedoensis Makino is a well-known native Chinese medicinal plant, which is reported to adapt to heavy metal pollution regions. In our preliminary studies, transcriptome sequencing was employed to identify global expression gene using a combined bioinformatics, and plenty of differential expression genes (DEGs) were also identified in V. yedoensis Makino responsive to cadmium (Cd) pollution. However, the relative molecular marker development was not expounded. In this study, we aimed to identify novel SSR (simple sequence repeat) and SNP (single-nucleotide polymorphism) markers in V. yedoensis Makino, as well as further study of the functional annotation, classification, and phylogenetic analysis of cyclotide family genes. Approximate 91.99 million high-quality clean reads were generated. 11176 simple sequence repeats (SSRs) were predicted in 9644 unigenes, of which, 1283 unigenes contained multiple SSRs. The SSRs consisted of 1732 dinucleotide motifs, 2733 trinucleotide motifs, 135 tetranucleotide motifs, 17 pentanucleotide motifs, and 13 hexanucleotide motifs. 163 motifs were detected among all of the SSRs identified. Moreover, 8250 SNP markers were detected in 1934 unigenes and 11328 SNP markers were detected in 2510 unigenes in VIYCd and VIYCK library respectively. Furthermore, 41 cyclotide family Unigenes in Violaceae were identified and categorized into six groups with the other species. This study firstly generated a valuable resource for SNP and SSR marker development studies of Violaceae transcriptome and thereby providing the basis for further studies on genomics and functional genomics in Violaceae.
\end{abstract}

KEYWORDS: Viola yedoensis Makino, SSRs, SNPs, cyclotide family

\section{INTRODUCTION}

Viola yedoensis Makino, an important native Chinese traditional medicine, is a small perennial plant with violet flowers distributed in China [1-4]. The dried whole plant (known as "Herba Violae") is an important constituent of the traditional medicine " $\mathrm{Zi}$ Hua Di Ding", which have been used traditionally to treat acute pyogenic infection, respiratory and inflammatory diseases, including bronchitis, hepatitis, swelling, boils and enteritis $[1,5]$. Recent studies have revealed that cyclotides are a group of plantderived peptides with unique structural properties, and almost all of cyclotides are isolated from Violaceae family [2]. Cyclotides have been reported to possess potent effect of anti-human immunod- eficiency virus and weak antibacterial activity and unmanageable toxicity $[3,5]$.

In recent years, the high-throughput sequencing technology (second-generation sequencing technology) for large scale transcriptome sequencing has dramatically improved the efficiency of genome annotation and gene discovery of non-model species, especially for Violaceae family [6-8]. However, there are not much research on $V$. yedoensis Makino, especially not enough for genomic study and functional gene identification. Simple sequence repeat (SSR) and single-nucleotide polymorphism (SNP) markers have been frequently used in the study of genetic identification and fingerprint mapping, and can be easily detected by the high-throughput sequencing technology [6]. Currently only few SSR 
and SNP markers were developed in $V$. yedoensis Makino. SSR has been widely used in the study of genetic identification and fingerprint mapping with the characteristics of high polymorphic information content, simple technology, and good reproducibility. SNP is the most abundant type of markers and is easy to develop and detect by high-throughput sequencing technology [6].

Recent studies have shown that $V$. yedoensis Makino is almost normally distributed in a Lead $(\mathrm{Pb}) /$ zinc $(\mathrm{Zn}) /$ cadmium (Cd) mine located in Sichuan Province, China. In our preliminary studies [9], Illumina paired-end sequencing technology was adopted to characterize the transcriptome of Violaceae through analysis of large-scale transcript sequences. The results would offer valuable sequence resource to Violaceae family and explore some unknown Cd-defensive strategies that possibly exist in the Viola species, as well as provide an efficient, inexpensive and reliable approach that can be readily adopted by researchers studying non-model organisms using transcriptome sequencing [9]. According to the results from the preliminary experiments, we characterize novel SSRs and SNPs of $V$. yedoensis Makino, and assess their functional annotation, classification, and phylogenetic analysis of cyclotide family genes.

\section{MATERIALS AND METHODS}

\section{Sample preparation and transcriptome sequencing}

V. yedoensis Makino were sown and transplanted in the nutrient solution (Table 1) as described previously. There were randomly divided to two groups, including control solution group (CK) and solution containing $2.5 \mathrm{mM} \mathrm{Cd}\left(\mathrm{NO}_{3}\right)_{2}$ group (Cd treatment). After $72 \mathrm{~h}$, whole plants were collected for total

Table 1 The detailed components of the nutrient solution.

\begin{tabular}{lr}
\hline Component & Concentration \\
\hline $\mathrm{Ca}\left(\mathrm{NO}_{3}\right)_{2} \cdot 4 \mathrm{H}_{2} \mathrm{O}$ & $4.0 \mathrm{mmol} / 1$ \\
$\mathrm{KNO}_{3}$ & $6.0 \mathrm{mmol} / 1$ \\
$\mathrm{NH}_{4} \mathrm{NO}_{3}$ & $1.0 \mathrm{mmol} / 1$ \\
$\mathrm{Fe} \cdot \mathrm{EDTA}_{3}$ & $100 \mu \mathrm{mol} / 1$ \\
$\mathrm{H}_{3} \mathrm{BO}_{3}$ & $1.0 \mu \mathrm{mol} / 1$ \\
$\mathrm{KH}_{2} \mathrm{PO}_{4}$ & $1.0 \mu \mathrm{mol} / 1$ \\
$\mathrm{MnSO}_{4}$ & $0.1 \mu \mathrm{mol} / 1$ \\
$\mathrm{CuSO}_{4} \cdot 7 \mathrm{H}_{2} \mathrm{O}$ & $0.1 \mu \mathrm{mol} / 1$ \\
$\mathrm{MgSO}_{4} \cdot 7 \mathrm{H}_{2} \mathrm{O}$ & $1.0 \mu \mathrm{mol} / 1$ \\
$\left(\mathrm{NH}_{4}\right)_{6} \mathrm{Mo}_{7} \mathrm{O}_{24}\left(\mathrm{H}_{2} \mathrm{O}\right)$ & $0.016 \mu \mathrm{mol} / 1$ \\
$\mathrm{ZnSO}_{4} \cdot 7 \mathrm{H}_{2} \mathrm{O}$ & $1.0 \mu \mathrm{mol} / 1$ \\
\hline
\end{tabular}

RNA extraction using TRIzol kit (Invitrogen, USA), and then were quantified, purified, and pooled for subsequent Illumina sequencing. In our preliminary studies [9], the de novo transcriptome assembly for gene identification, analysis, functional annotation and classification were accessed. Based on this, in the present study, we determine to further identify novel SSR and SNP markers and cyclotide family unigenes in $V$. yedoensis Makino responsive to cadmium (Cd) pollution.

\section{SSR prediction and SNP detection}

Mining of the SSRs present in the assembled unigenes of Violaceae was performed as described previously [10]. Briefly, five categories of SSRs, including those with dinucleotide, trinucleotide, tetranucleotide, pentanucleotide, and hexanucleotide motifs, were classified. For each category of SSR, the minimum number of contiguous repeat units is five. Statistical analysis was used to investigate the number of SSRs with each type of motif and the distribution of the lengths of repeat units. In addition, SNPs is also identified mentioned below: we firstly used Simple Object Access Protocol (SOAP) to map the reads sequences to the identified unigene, and put pair-end and single-end comparison results together, duplicated reads and multi-mapped reads were also filtered out, results were sorted according to the transcript and coordinate position, then we used SOAPsnp [11] to SNP calling for the sorted results, parameters were set as follows: "seed Length" 30, "minLength" 50, "minInsert" 100, "maxInsert" 1000, "misMatch" 3, "ASCII" "!". In order to get the high quality SNPs, the quantity and quality $(\geqslant 20)$, the sequencing depth $(\geqslant 2)$, and the SNP spacing $(\geqslant 5)$ were also selected to filter out the results. SSRs predicted in silico were validated by PCR amplification. The products were separated on $1.5 \%$ agarose gels and visualized using Goldview Nucleic Acid Stain.

\section{Phylogenetic analysis and RT-PCR validation of cyclotide family unigenes}

Hidden Markov Model (HMM) profile of cyclotide family domain (PF03784) downloaded from Protein family (Pfam; http://pfam.xfam.org) was exploited for the identification of the cyclotide family genes from Violaceae assembly transcriptome with HMMER (v 2.3.2) [12]. Conserved sequences of cyclotide family was extracted from the HMM profile by the HMMER software [12], and then was adopted to query the Violaceae assembly transcriptome. Searching parameters were followings: 
Table 2 The primers for identifing the cyclotide family unique genes.

\begin{tabular}{|c|c|c|}
\hline Unique gene & Primer sequence & Size \\
\hline \multirow{2}{*}{ comp109420_c0 Vya15 } & 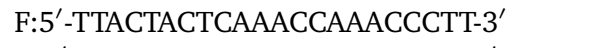 & \multirow{2}{*}{$273 \mathrm{bp}$} \\
\hline & R:5'-TTCGCTTCAGTTCAATCCTAC-3' & \\
\hline \multirow{2}{*}{ comp108309_c0 Vya10 } & F:5'-GTCATGTAACGATCAGT-3' & \multirow{2}{*}{$343 \mathrm{bp}$} \\
\hline & R:5'-GGAGATCTTTGTAGAAT-3' & \\
\hline \multirow{2}{*}{ comp109293_c0 Vya14 } & F:5'-TGCAGCTCCTTGTACTGA-3' & \multirow{2}{*}{ 398bp } \\
\hline & R:5'-CAAAAGCCCTTCTTCATA-3' & \\
\hline \multirow{2}{*}{ comp102243_c0 Vya1 } & F:5'-CGCAACAATAGATAGACA-3' & \multirow{2}{*}{$343 b p$} \\
\hline & R:5'-TCACATCCAATACAACAG-3' & \\
\hline \multirow{2}{*}{ comp403622_c0 Vya26 } & F:5' - ACCAAAACCATCGTCTC-3' & \multirow{2}{*}{$252 \mathrm{bp}$} \\
\hline & R:5'-AGACAACAAGCGAAAC-3' & \\
\hline \multirow{2}{*}{ comp87919_c0 Vya37 } & F:5'-ACCAAAACCATCGTCTCAAACCCGG-3' & \multirow{2}{*}{$280 \mathrm{bp}$} \\
\hline & R:5'-CCGGGTTTGAGACGATGGTTTTGGT-3' & \\
\hline \multirow{2}{*}{ comp109209_c0 Vya13 } & F:5'-TGGAACGAAGGGAACA-3' & \multirow{2}{*}{$358 b p$} \\
\hline & R:5'-AGACTTGCGTCGGTGG-3' & \\
\hline \multirow{2}{*}{ comp120436_c0 Vya21 } & F:5'-CATTACTACTCAAACCAAACCCTT-3' & \multirow{2}{*}{ 305bp } \\
\hline & R:5'-TTCGCTTCAGTTCAATCCTACCTC-3' & \\
\hline \multirow{2}{*}{ comp4648_c0 Vya27 } & F:5'-GTTTATCGACTTGTGCAG-3' & \multirow{2}{*}{ 388bp } \\
\hline & R:5'-ATGGAAAAGAAGAAGAAG-3' & \\
\hline \multirow{2}{*}{ comp104964_c0 Vya6 } & F:5'-ATCCAACTACCCAACA-3' & \multirow{2}{*}{ 394bp } \\
\hline & R:5'-GCCATGATTTAAGCGT-3' & \\
\hline \multirow{2}{*}{ comp99755_c1 Vya41 } & F:5'-GAGTTCTTGTAGCAAACT-3' & \multirow{2}{*}{ 419bp } \\
\hline & R:5'-AAGTCACATACCTTACCG-3' & \\
\hline
\end{tabular}

BLASTp, $E=1 \mathrm{e}-10$, and other parameters were defaulted. All non-redundant hits with expected values less than 1.0 were collected, and then the identified Violaceae cyclotide family unigenes were first aligned using CLUSTAL X version 2.0 [13]. Subsequently, based on the nucleotide sequence alignments, MEGA4.0 software was used to generate phylogenetic trees of Violaceae Unigenes using the neighbor-joining approach [14]. The phylogenetic trees of cyclotide family were furnished with 1000 pseudoreplicate bootstrap values at each node, with the similar cyclotide family from the Melicytus ramiflorus (Mra), Viola baoshanensis (Vba) and Viola odorata (Voa). The accession numbers for the sequences of cyclotide family is named Vya1Vya41 in (Supplementary Table S1). In addition, 13 assembled unigenes were randomly selected from four cyclotide family groups for RT-PCR validation. Total RNA from $V$. yedoensis leaves was reversetranscribed by using SuperScript III Reverse Transcriptase (Invitrogen) and oligo(dT)18. Forward (Fwd) and reverse (Rev) primers were designed using Primer3. The sequences of primers used for validation of assembled $V$. yedoensis cyclotide family unigenes were listed in Table 2.
Table 3 SSR analysis of the $V$. yedoensis transcriptome.

\begin{tabular}{lcc}
\hline Unit size & No. of SSRs & Percent \\
\hline 1 & 6546 & 58.57 \\
2 & 1732 & 15.50 \\
3 & 2733 & 24.45 \\
4 & 135 & 1.21 \\
5 & 17 & 0.15 \\
6 & 13 & 0.12 \\
\hline
\end{tabular}

\section{RESULTS AND DISCUSSION}

\section{SSR analysis of the Violaceae transcriptome}

In our preliminary studies, the de novo transcriptome assembly for gene identification, analysis, functional annotation and classification were accessed [9]. However, the molecular marker development was not expounded. SSR markers, also known as microsatellites, are repeating DNA sequences of 2-6 base pairs, which are widely used as molecular markers for genetic mapping and to analyze species diversity. Approximate 91.99 million high-quality clean reads were generated. Then, 11176 SSRs were predicted in 9644 unigenes, of which, 1283 unigenes contained multiple SSRs. The SSRs included 1732 (15.5\%) dinucleotide motifs, 2733 (24.45\%) trinucleotide motifs, 135 (1.21\%) tetranucleotide motifs, $17(0.15 \%)$ pentanucleotide 
ScienceAsia 46 (2020)

Table 4 The distribution of the number of repeat units in all SSRs.

\begin{tabular}{|c|c|c|c|c|c|c|c|c|c|c|c|c|c|c|c|c|c|c|c|c|c|c|c|c|}
\hline Repeat & 5 & 6 & 7 & 8 & 9 & 10 & 11 & 12 & All & Repeat & 5 & 67 & 78 & $\begin{array}{ll}9 & 1\end{array}$ & 1011 & $11 \quad 12$ & All & Repeat & 5 & 6 & 78 & 9 & 1011 & 12 All \\
\hline AC & - & 45 & 19 & 11 & 6 & 6 & 11 & & 98 & AAAC & 2 & & & & & & 2 & AAAGC & & & 1 & & & 1 \\
\hline AG & - & 131 & 64 & 40 & 22 & 18 & 12 & 1 & 288 & AAAG & 3 & & & & & & 3 & AAGCC & 1 & & & & & 1 \\
\hline AT & - & 98 & 59 & 27 & 18 & 10 & 10 & & 222 & AAAT & 2 & & & & & & 2 & AATAA & 1 & & & & & 1 \\
\hline CA & - & 35 & 18 & 16 & 7 & 6 & 3 & & 85 & AACC & & 1 & & & & & 1 & AATCA & 1 & & & & & 1 \\
\hline CG & - & 2 & 1 & & & & & & 3 & AAGA & 1 & 1 & & & & & 2 & AGAAT & 1 & & & & & 1 \\
\hline CT & - & 105 & 44 & 24 & 21 & 15 & 4 & & 213 & AATA & 4 & & & & & & 4 & AGCTG & 1 & & & & & 1 \\
\hline GA & - & 76 & 49 & 21 & 22 & 18 & 17 & 2 & 205 & AATC & 1 & & & & & & 1 & AGGCT & 1 & & & & & 1 \\
\hline GC & - & 8 & 1 & & & & & & 9 & AATG & 1 & & & & & & 1 & ATCAC & 1 & & & & & 1 \\
\hline GT & - & 49 & 19 & 12 & 12 & 16 & 10 & & 118 & AATT & 2 & & & & & & 2 & CСATC & 1 & & & & & 1 \\
\hline TA & - & 91 & 35 & 37 & 21 & 19 & 5 & & 208 & ACAA & 1 & & & & & & 1 & CGATG & 1 & & & & & 1 \\
\hline TC & - & 84 & 38 & 21 & 12 & 6 & 9 & & 170 & ACAT & 1 & & & & & & 1 & CGGAA & & 1 & & & & 1 \\
\hline TG & - & 44 & 33 & 15 & 7 & 5 & 9 & & 113 & ACCA & 1 & & & & & & 1 & GAAAC & 1 & & & & & 1 \\
\hline AAC & 27 & 8 & 11 & & & & & & 46 & ACTC & 1 & & & & & & 1 & GAAGA & & & 1 & & & 1 \\
\hline AAG & 51 & 25 & 15 & & & & & & 91 & AGAA & 1 & 1 & & & & & 2 & GGTTG & 1 & & & & & 1 \\
\hline AAT & 26 & 11 & 10 & & & & & & 47 & AGGA & 1 & & & & & & 1 & TGACC & 1 & & & & & 1 \\
\hline ACA & 31 & 5 & 2 & & & & & & 39 & AGGG & 1 & & & & & & 1 & TGAGC & 1 & & & & & 1 \\
\hline ACC & 23 & 10 & & & & & & & 33 & ATAA & 4 & 1 & & & & & 5 & TTGGT & 1 & & & & & 1 \\
\hline ACG & 1 & 1 & 1 & & & & & & 3 & ATAC & 4 & & & & & & 4 & ACGCCA & & 1 & & & & 1 \\
\hline ACT & 6 & 2 & 1 & & & & & & 9 & ATCA & & 1 & & & & & 1 & ACGGCT & & 1 & & & & 1 \\
\hline AGA & 34 & 21 & 11 & & & & & & 66 & ATCC & 1 & & & & & & 1 & ATCCCT & 1 & & & & & 1 \\
\hline AGC & 59 & 16 & 3 & 1 & & & & & 79 & ATGC & 1 & & & & & & 1 & CACAGT & & 1 & & & & 1 \\
\hline AGG & 46 & 18 & 9 & & & & & & 73 & ATGG & & 1 & & & & & 1 & CAGGAA & 1 & & & & & 1 \\
\hline AGT & 12 & 2 & & & & & & & 14 & ATGT & 1 & & & & & & 1 & CCCGGA & & 1 & & & & 1 \\
\hline ATA & 15 & 5 & 8 & 1 & & & & & 29 & ATTA & 4 & & & & & & 4 & CCGGGA & & 1 & & & & 1 \\
\hline ATC & 38 & 13 & 5 & & & & & & 56 & ATTT & 2 & & & & & & 2 & GGGAGA & & 1 & & & & 1 \\
\hline ATG & 30 & 15 & 4 & & & & & & 49 & CAAA & 2 & & & & & & 2 & GTAGGG & & 1 & & & & 1 \\
\hline ATT & 23 & 9 & 5 & & & & & & 37 & CAAT & 1 & & & & & & 1 & GTGAGC & 1 & & & & & 1 \\
\hline CAA & 45 & 11 & 7 & & & & & & 63 & СACT & 1 & 1 & & & & & 2 & TGGTTA & 1 & & & & & 1 \\
\hline CAC & 29 & 11 & 3 & & & & & & 43 & CATA & 1 & & & & & & 1 & ТTCTCC & & & & & 1 & 1 \\
\hline CAG & 59 & 23 & 7 & & & & & & 89 & CATC & 1 & & & & & & 1 & TTGACA & & 1 & & & & \\
\hline CAT & 26 & 12 & 3 & & & & & & 41 & CCAA & 1 & & & & & & 1 & & & & & & & \\
\hline CCA & 35 & 16 & 4 & 1 & & & & & 56 & CCAT & & 1 & & & & & 1 & & & & & & & \\
\hline CCG & 10 & 3 & & 1 & & & & & 14 & CСТC & 1 & & & & & & 1 & & & & & & & \\
\hline СCT & 41 & 19 & 2 & 1 & & & & & 63 & CGTC & 1 & & & & & & 1 & & & & & & & \\
\hline CGA & 5 & 4 & 1 & & & & & & 10 & CTCA & 1 & & & & & & 1 & & & & & & & \\
\hline CGC & 7 & 4 & 2 & 1 & & & & & 14 & CTCC & 2 & & & & & & 2 & & & & & & & \\
\hline CGG & 7 & 5 & & & & & & & 12 & CTGA & 1 & & & & & & 1 & & & & & & & \\
\hline CGT & 5 & & 1 & & & & & & 6 & CTTC & 2 & 1 & & & & & 3 & & & & & & & \\
\hline CTA & 5 & & 2 & & & & & & 7 & CTTT & 1 & & & & & & 1 & & & & & & & \\
\hline CTC & 48 & 15 & 5 & & & & & & 68 & GAAA & 2 & 1 & & & & & 3 & & & & & & & \\
\hline CTG & 42 & 9 & 5 & & & & & & 56 & GAAG & 4 & & & & & & 4 & & & & & & & \\
\hline CTT & 32 & 14 & 6 & 1 & & & & & 53 & GACA & & 1 & & & & & 1 & & & & & & & \\
\hline GAA & 59 & 23 & 13 & 1 & & & & & 96 & GAGG & 1 & & & & & & 1 & & & & & & & \\
\hline GAC & 4 & 3 & & 1 & & & & & 8 & GATG & 3 & 1 & & & & & 4 & & & & & & & \\
\hline GAG & 77 & 36 & 10 & & & & & & 124 & GCAT & 1 & & & & & & 1 & & & & & & & \\
\hline GAT & 36 & 13 & 9 & & & & & & 58 & GCCT & 1 & & & & & & 1 & & & & & & & \\
\hline GCA & 41 & 21 & 11 & & & & & & 73 & GTGA & 1 & & & & & & 1 & & & & & & & \\
\hline GCC & 5 & 1 & 2 & & & & & & 8 & GTTA & 1 & & & & & & 1 & & & & & & & \\
\hline GCG & 6 & 2 & & & & & & & 8 & TAAA & 3 & & & & & & 3 & & & & & & & \\
\hline GCT & 42 & 16 & 8 & & & & & & 66 & TAAT & 3 & 1 & & & & & 4 & & & & & & & \\
\hline GGA & 80 & 18 & 5 & 2 & & & & & 105 & TACC & 1 & & & & & & 1 & & & & & & & \\
\hline GGC & 22 & 7 & 2 & & & & & & 31 & TATG & 1 & & & & & & 1 & & & & & & & \\
\hline GGT & 51 & 13 & 7 & 3 & & & & & 74 & TATT & 4 & & & & & & 4 & & & & & & & \\
\hline GTA & 6 & 5 & 1 & 1 & & & & & 13 & TCAA & 1 & & & & & & 1 & & & & & & & \\
\hline GTC & 8 & & 1 & & & & & & 9 & TCAC & 1 & & & & & & 1 & & & & & & & \\
\hline GTG & 35 & 12 & 5 & & & & & & 52 & TCCA & 1 & & & & & & 1 & & & & & & & \\
\hline GTT & 28 & 7 & 10 & & & & & & 45 & TCCC & 1 & & & & & & 1 & & & & & & & \\
\hline TAA & 12 & 7 & 11 & & & & & & 30 & TCCG & & 1 & & & & & 1 & & & & & & & \\
\hline TAC & 13 & 2 & & & & & & & 15 & TCCT & 1 & & & & & & 1 & & & & & & & \\
\hline TAG & 6 & 4 & 1 & & & & & & 11 & TCTT & 3 & & & & & & 3 & & & & & & & \\
\hline TAT & 20 & 6 & 5 & & & & & & 31 & TGCA & 1 & & & & & & 1 & & & & & & & \\
\hline TCA & 44 & 6 & 5 & & 1 & & & & 56 & TGGA & 2 & & & & & & 2 & & & & & & & \\
\hline TCC & 45 & 22 & 5 & & & & & & 72 & TGGC & 1 & & & & & & 1 & & & & & & & \\
\hline TCG & 10 & 3 & 2 & & & & & & 15 & TGTA & 3 & & & & & & 3 & & & & & & & \\
\hline TCT & 36 & 17 & 7 & & & & & & 60 & TTAA & 4 & & & & & & 4 & & & & & & & \\
\hline TGA & 38 & 17 & 4 & 1 & & & & & 60 & TTAT & 1 & & & & & & 1 & & & & & & & \\
\hline TGC & 47 & 19 & 4 & & & & & & 70 & TTCA & 4 & & & & & & 4 & & & & & & & \\
\hline TGG & 45 & 19 & 6 & 1 & & & & & 71 & TTCC & & & & & 1 & & 1 & & & & & & & \\
\hline TGT & 15 & 6 & 5 & 1 & & & & & 27 & TTCT & 4 & & & & & & 4 & & & & & & & \\
\hline TTA & 24 & 8 & 4 & 1 & & & & & 37 & TTTA & 4 & & & & & & 4 & & & & & & & \\
\hline TTC & 40 & 13 & 9 & & & & & & 62 & TTTC & 1 & & & & & & 1 & & & & & & & \\
\hline TTG & 58 & 14 & 8 & & & & & & 80 & TTTG & 5 & & & & & & 5 & & & & & & & \\
\hline
\end{tabular}


motifs, and $13(0.12 \%)$ hexanucleotide motifs (Table 3). Furthermore, 163 motifs were detected among all of the SSRs identified. The most abundant SSR motif was GA/AG/TA/AT (9, 23 SSRs), followed by AAG/AGA/TTC/TCT/CTT/GAA/GAG (7, 22 SSRs), and then AC/CA/TC/CT (566 SSRs). The distribution of the number of repeat units in all SSRs was also investigated (Table 4). The result revealed that most SSRs contained fewer than 12 repeat sequences, and no SSRs with more than 20 repeat sequences were observed. Here, we predicted 6295 SSRs from the assembled unigene library of Violaceae. These SSRs will likely be of value for genetic analyses of species in the genus Violaceae and other related non-model plants. We selected primer S1 and primer S2 to check the PCR amplification and polymorphism of V. yedoensis samples, for example, $11 \mathrm{~V}$. yedoensis samples with the SSR primers developed in this study were detected, and gel pictures of results were shown in Fig. 1. Our data suggested that SSRs locus predicted in silico could be very useful resources for SSR marker development of $V$. yedoensis.

\section{SNP analysis of the Violaceae transcriptome}

Furthermore, application of SNP marker technology has potential to improve plant genetics, and facilitate faster release of plant hybrids to the market place. The SNP usually occurs in non-coding regions more frequently than in coding regions or, in general, where natural selection is acting and fixating the allele of the SNP that constitutes the most favorable genetic adaptation [15]. Other factors, like genetic recombination and mutation rate, can also determine SNP density [16]. Here, in total, 8250 SNP markers were detected in 1934 unigenes and 11328 SNP markers were detected in 2510 unigenes in VIYCd and VIYCK library, respectively. Moreover, 83 homogenous SNPs were detected in 76 unigenes and 55 SNP markers were detected in 51 unigenes in two libraries, respectively (Fig. 2, Supplementary Tables S2 and S3).

\section{Phylogenetic analysis of cyclotide family genes}

Most interestingly, 41 cyclotide family genes were identified and then annotated based on assembly transcriptome, and 11 cyclotide family unique randomly selected from six groups (Fig. 3) were validated by RT-PCR. Of these, all of them could successfully yield amplicons, thereby provided evidence for the quality validation of our assembled cyclotide family Unigenes. In addition, Gene Ontology (GO) annotation showed that cyclotide family genes were involved in defense response and DNA repair/DNA metabolic process. Based on the Phylogenetic analysis of cyclotide family unigenes, we classified these cyclotide family into 6 groups, of which, comp109420 (Vya15) contained MADS-box domain by KOG classify which is clustered in group V (Fig. 4, Supplementary Table S1). It is interesting that group $\mathrm{V}$ was only found in our research.

Cyclotides are a novel family of plant-derived defense peptides that are biosynthetically produced via the processing of cyclotide precursor (CP) proteins containing one, two or three cyclotide domains. Cyclotide sequence analyses indicated that the cDNA clones encoded a variety of Möbius and bracelet cyclotides, which were involved in the known bioactivities of cyclotides, and also might play a previously unreported role in mediating the metal tolerance of $V$. baoshanensis. In addition, Cdresponsive gene $\mathrm{Vb} 40$ was identified, which could deduce a novel cysteine-rich mini-protein and could improve copper $(\mathrm{Cu})$ tolerance in hosted yeast, indicating that these species-specific genes possibly functioned in V. baoshanensis heavy metal (Cd) tolerance [17]. In this study, 41 cyclotide family unigenes in Violaceae were annotated based on six known databases and categorized into six groups. Based on GO annotation, we demonstrated that these cyclotide family genes were probably involved in mediating the metal tolerance in Violaceae.

\section{CONCLUSION}

In our preliminary studies, we determined the de novo transcriptome assembly for gene identification, analysis, functional annotation and classification using Illumina paired-end sequencing technology for Violaceae transcriptome de novo sequencing and assembly without reference genome, and plenty of differential gene expression levels were measured in $V$. yedoensis Makino responsive to cadmium (Cd) pollution. However, the relative molecular marker development was not expounded. Based on this, in the present study, we determine to identify novel SSR and SNP markers in V. yedoensis Makino, and also study the functional annotation, classification, and phylogenetic analysis of cyclotide family genes. Taken together, such large number of sequences and deep depth of coverage can provide sufficient transcriptomic sequence information for discovering novel genes. Additionally, thousands of SSR markers produced in this study will enable genetic linkage mapping construction and gene-based association studies. The results demonstrated that Illumina paired end sequencing can be used as a fast 


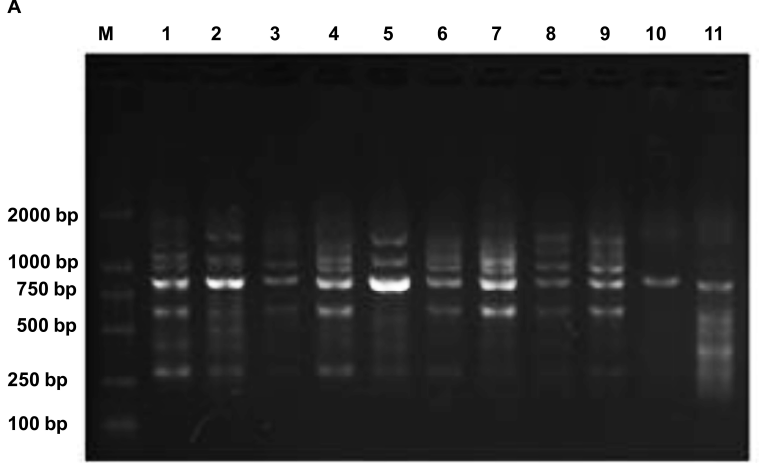

B

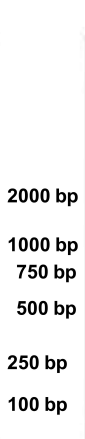

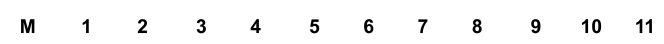

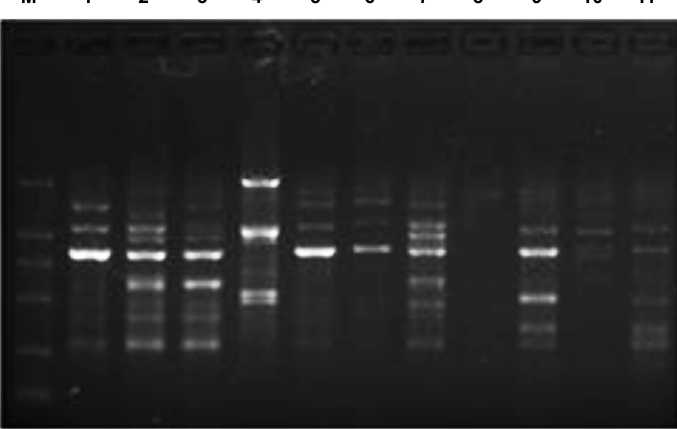

Fig. 1 PCR amplification of $11 \mathrm{~V}$. yedoensis samples with the SSR primers developed in this study. (A) Primer S1 amplification results. (B) Primer S2 amplification results. Lane 1, GuLin of Luzhou in Sichuan; Lane 2, XuYong of Luzhou in Sichuan; Lane 3, NaXi of Luzhou in Sichuan; Lane 4, BanQiao of Zigong in Sichuan; Lane 5, BeiBei of Chongqing; Lane 6, HeChuan of Chongqing; Lane 7, YongChuan of Chongqing; Lane 8, WenHai of Lijiang in Yunnan; Lane 9, WeiYuan of Neijiang in Sichuan; Lane 10, XinWen of Yibing in Sichuan; Lane 11, GongXian of Yibing in Sichuan; Lane M, molecular marker DL2000.

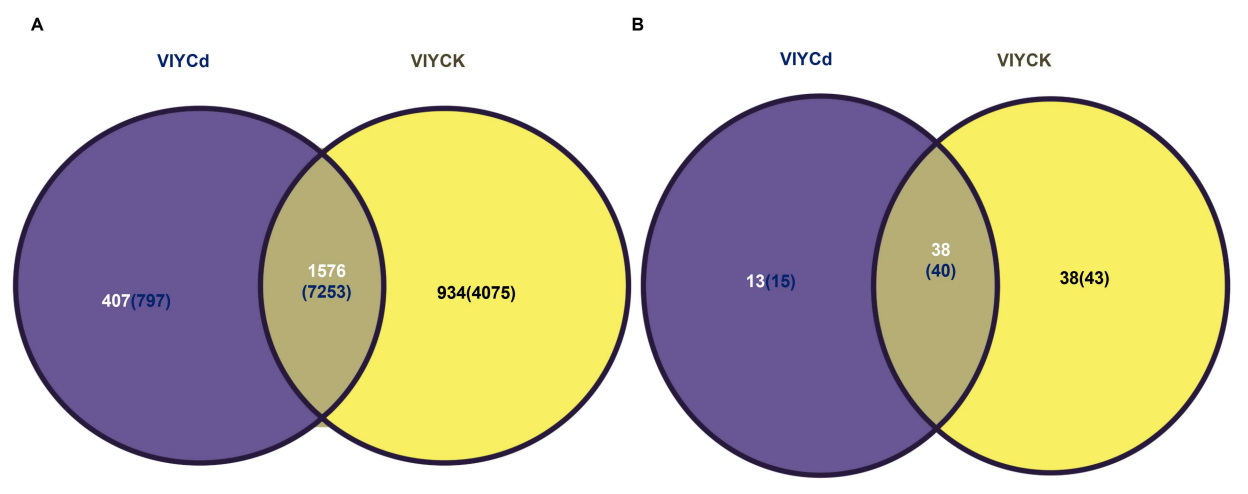

Fig. 2 The total and homogenous SNPs identified in two libraries. (A) Total SNPs were identified in two libraries. Violet and yellow color showed SNPs in CK and Cd libraries, respectively, 13927 SNPs were common distributed in both libraries. (B) Homogenous SNPs were identified in two libraries. Violet and yellow color showed homogenous SNPs in CK and Cd libraries, respectively, 321 homogenous SNPs were common distributed in both libraries.

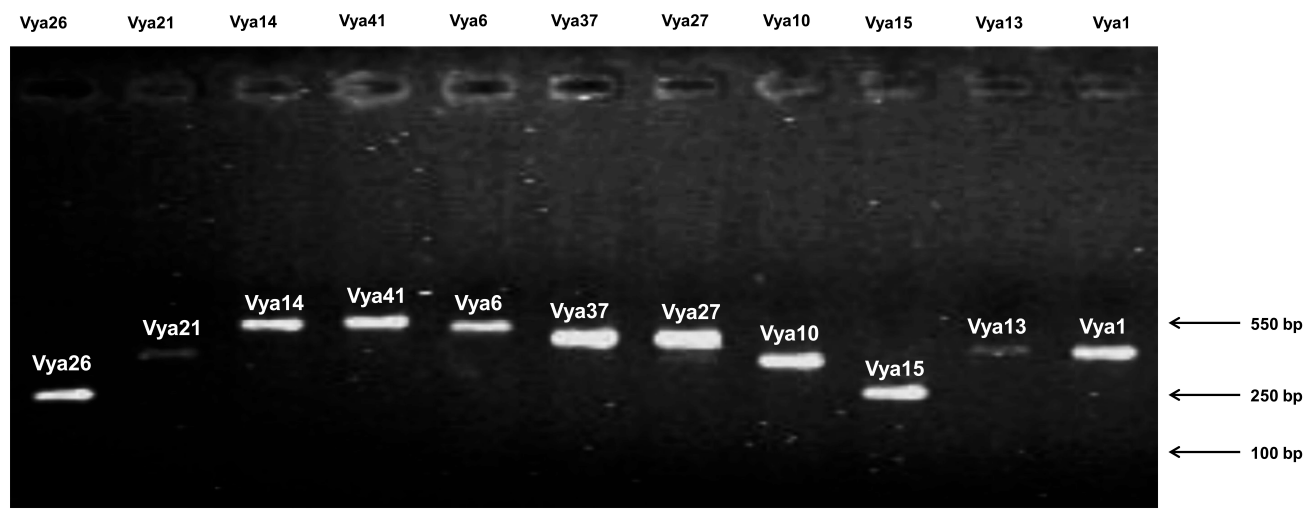

Fig. 3 Cyclotide family unique randomly selected from six groups validated by RT-PCR. The CDS length is in range from $252 \mathrm{bp}$ to $419 \mathrm{bp}$ in these 11 Unigenes. 


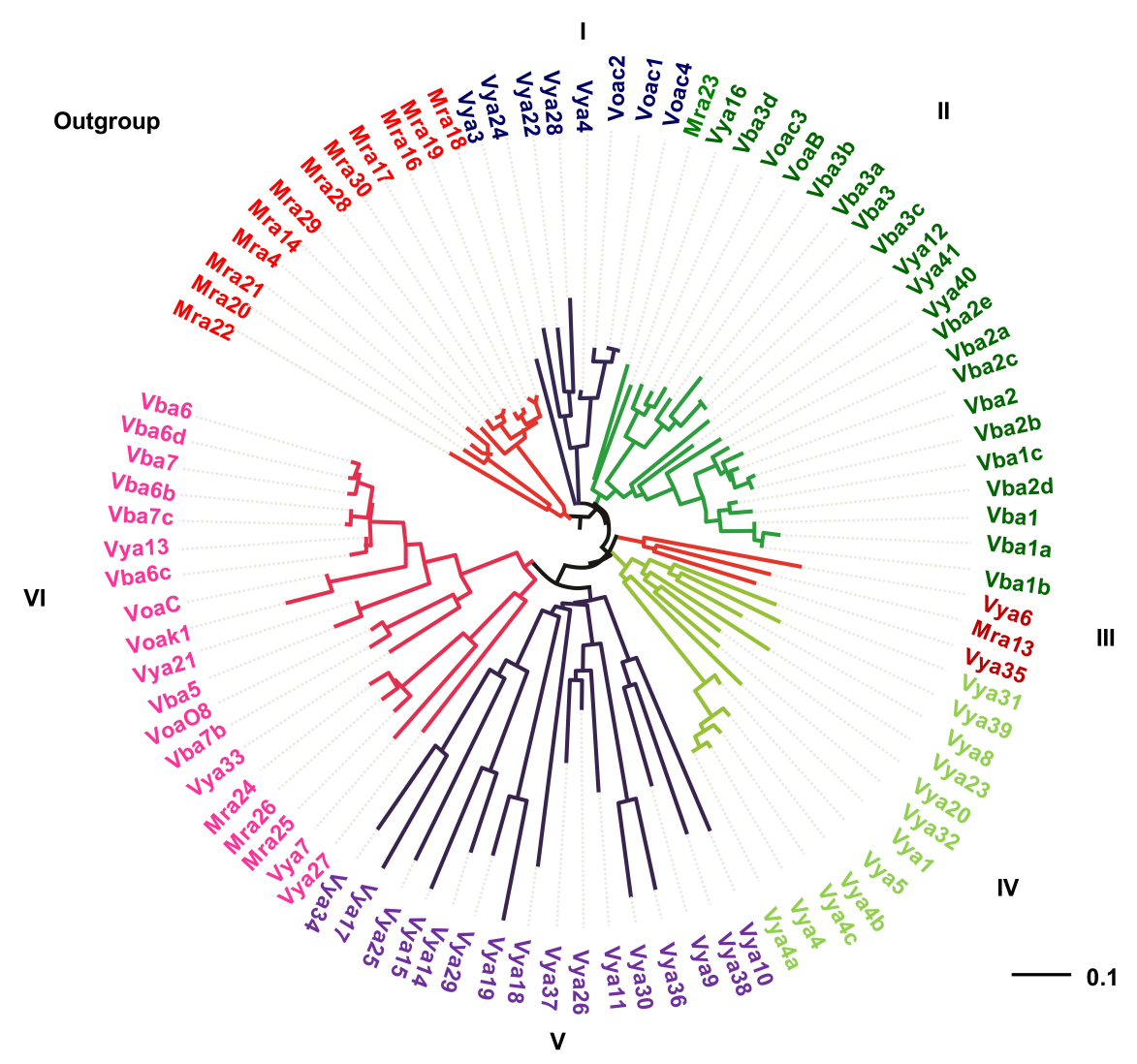

Fig. 4 The phylogenetic tree analysis of cyclotide family Unigenes based on transcriptome assembly and annotation. The phylogenetic tree of cyclotide family Unigenes in multiple plants were constructed using the neighbor-joining method, including Melicytus ramiflorus (Mra), Viola baoshanensis (Vba) and Viola odorata (Voa).

and cost-effective approach to the gene discovery and molecular marker development for non-model organism, especially those with large genome.

Supplementary data: Supplementary Tables S1, S2 and S3 are available upon request from the authors.

$\mathrm{S} 1$. Assembly and annotation of cyclotide genes in V. yedoensis based on the database.

S2. Total SNPs identified in unigenes of two libraries.

S3. Homogenous SNPs identified in unigenes of two libraries.

Acknowledgements: This work was supported by the National Natural Science Foundation of China (81800434, 81001700), Grant of Sichuan Province Science and Technology Agency Grant (14JC0137, 2018JY017 and 2019YJ0487), Grant of Chengdu Municipal Science and Technology Bureau of Sichuan Province (2019-YFYF-00167-SN), and the Foundation of Luzhou Municipal Science and Technology Bureau of Sichuan Province (2017LZXNYD-T05, 2016LZXNYD-J24 and 2019LZXNYDJ49).

\section{REFERENCES}

1. Li W, Xie JY, Li H, Zhang YY, Cao J, Cheng ZH, Chen DF (2012) Viola yedoensis liposoluble fraction ameliorates lipopolysaccharide-induced acute lung injury in mice. Am J Chin Med 40, 1007-1018.

2. Xie C, Veitch NC, Houghton PJ, Simmonds MS (2003) Flavone C-glycosides from Viola yedoensis MAKINO. Chem Pharm Bull (Tokyo) 51, 1204-1207.

3. Wang CK, Colgrave ML, Gustafson KR, Ireland DC, Goransson U, Craik DJ (2008) Anti-HIV cyclotides from the Chinese medicinal herb Viola yedoensis. $J$ Nat Prod 71, 47-52.

4. Zeng HR, Wang B, Zhao Z, Zhang Q, Liang MY, Yao YQ, Bian K, Zhang WR (2016) Effects of Viola yedoensis Makino anti-itching compound on degranulation and cytokine generation in RBL-2H3 mast cells. $J$ Ethnopharmacol 189, 132-138.

5. Jeong YH, Oh YC, Cho WK, Shin H, Lee KY, Ma JY (2016) Anti-inflammatory effects of Viola yedoensis and the application of cell extraction methods for investigating bioactive constituents in macrophages. BMC Complement Altern Med 16, ID 180. 
6. Zou D, Chen X, Zou D (2013) Sequencing, de novo assembly, annotation and SSR and SNP detection of sabaigrass (Eulaliopsis binata) transcriptome. Genomics 102, 57-62.

7. Mora-Ortiz M, Swain MT, Vickers MJ, Hegarty MJ, Kelly R, Smith LM, Skøt L (2016) De-novo transcriptome assembly for gene identification, analysis, annotation, and molecular marker discovery in Onobrychis viciifolia. BMC Genomics 17, 756.

8. Liang M, Yang X, Li H, Su S, Yi H, Chai L, Deng X (2015) De novo transcriptome assembly of pummelo and molecular marker development. PLoS One 10, e0120615.

9. Gao J, Luo M, Zhu Y, He Y, Wang Q, Zhang C (2015) Transcriptome sequencing and differential gene expression analysis in Viola yedoensis Makino (Fam. Violaceae) responsive to cadmium (Cd) pollution. Biochem Biophys Res Commun 459, 60-65.

10. Zhang J, Liang S, Duan J, Wang J, Chen S, Cheng Z, Zhang Q, Liang X, et al (2012) De novo assembly and characterisation of the transcriptome during seed development, and generation of genic-SSR markers in Peanut (Arachis hypogaea L.). BMC Genomics 13, ID 90.
11. Li R, Li Y, Fang X, Yang H, Wang J, Kristiansen K, Wang $\mathrm{J}$ (2009) SNP detection for massively parallel wholegenome resequencing. Genome Res 19, 1124-1132.

12. Eddy SR (1998) Profile hidden Markov models. Bioinformatics 14, 755-763.

13. Larkin MA, Blackshields G, Brown NP, Chenna R, McGettigan PA, McWilliam H, Valentin F, Wallace IM, et al (2007) Clustal W and Clustal X version 2.0. Bioinformatics 23, 2947-2948.

14. Tamura K, Dudley J, Nei M, Kumar S (2007) MEGA4: molecular evolutionary genetics analysis (MEGA) software version 4.0. Mol Biol Evol 24, 1596-1599.

15. Barreiro LB, Laval G, Quach H, Patin E, QuintanaMurci L (2008) Natural selection has driven population differentiation in modern humans. Nat Genet 40, 340-345.

16. Rafalski A (2002) Applications of single nucleotide polymorphisms in crop genetics. Curr Opin Plant Biol 5, 94-100.

17. Zhang J, Hu M, Li JT, Guan JP, Yang B, Shu WS, Liao B (2009) A transcriptional profile of metallophyte Viola baoshanensis involved in general and species-specific cadmium-defense mechanisms. J Plant Physiol 166, 862-870. 